\title{
PARTIALLY ORDERED GROUPS OF THE SECOND AND THIRD KINDS ${ }^{1}$
}

\author{
A. H. CLIFFORD
}

1. Introduction. Let $G$ be both a group and a partially ordered set. An element $a$ of $G$ is called a left [right] conserver if

$$
x \leqq y(x, y \in G) \Rightarrow a x \leqq a y[x a \leqq y a]
$$

and a left [right] inverter if

$$
x \leqq y(x, y \in G) \Rightarrow a x \geqq a y[x a \geqq y a] .
$$

We shall call an element of $G$ a conserver [inverter] if it is both a left and a right conserver [inverter].

If every element of $G$ is a conserver, then $G$ is a partially ordered group (abbreviated "po-group") in the usual sense; we shall also say that $G$ is a po-group of the first kind. If every element of $G$ is a conserver or an inverter, and not every element of $G$ is a conserver, then we shall call $G$ a po-group of the second kind. A familiar example is the multiplicative group of all nonzero real numbers with the usual ordering. The stipulation that not every element of $G$ is a conserver excludes the possibility that $G$ be trivially ordered, and it is then clear that no element of $G$ can be both a conserver and an inverter.

The structure of totally ordered groups ("o-groups") of the second kind has been reduced to that of o-groups of the first kind by J. A. H. Shepperd [1]. (What he calls a "betweenness group" is either an o-group of the first or second kinds, or a finite group of order 4.) The first main result of the present note (Theorem 1) is an extension of Shepperd's result from o-groups to po-groups. The proof has also been simplified by avoiding reference to the betweenness relation.

Totally ordered semigroups ("o-semigroups") of the second kind have been considered by the author [2] in the commutative case, and by J. Gilder [3] and K. Keimel [4] in general. Following Gilder's terminology, we define a po-group of the third kind to be a group $G$ endowed with a nontrivial partial order, such that each element of $G$ is either a left conserver or a left inverter, and also either a right conserver or a right inverter, and such that $G$ contains an element which conserves on one side and inverts on the other. Theorem 2 gives a reduction of these to po-groups of the first kind.

Received by the editors December 18, 1964.

1 This paper was prepared with the support of a National Science Foundation Grant (GP 1791) to Tulane University. 
Added in proof. The author regrets that he was not aware at the time of writing this paper that a result equivalent to Theorem 1 below had been obtained by J. F. Andrus and A. T. Butson [5] for a connected po-group of the second kind. The two approaches are also different, however, that the equivalence of the results is not apparent. Their subgroup $S_{0}$ is the (directed) po-subgroup of my subgroup $H$ generated by its positive cone $H_{+}$. My subset $I_{-}$of $G \backslash H$ is the union of those cosets of $S_{0}$ in $G$ which belong to their subset $T_{0}$ of the factor group $G / S_{0}$. Removing their requirement of connectedness actually simplifies more than it complicates. Thus the six properties (i)-(vi) which $T_{0}$ must have in their Theorem 5 reduce to (i), (iv), (v), and the requirement that there exists a subgroup $H$ of index 2 in $P$ ( $\left.=G / S_{0}\right)$ such that $T_{0} \leqq G \backslash H$. (Incidently, (v) should read " $a+T_{0}$ $=T_{a}$ ") This is an immediate consequence of Theorem 1 below, in the case $H_{+}=0$.

2. Partially ordered groups of the second kind. We denote the identity element of $G$ by $e$, and set

$$
G_{+}=\{a \in G: a \geqq e\}, \quad G_{-}=\{a \in G: a \leqq e\} .
$$

For any subset $A$ of $G$, we let $A_{+}=A \cap G_{+}, A_{-}=A \cap G_{-}$, and $A^{-1}=\left\{a^{-1}: a \in A\right\}$. A (possibly empty) subset $A$ of $G$ is called an upper [lower] class in $G$ if $a \in A, x \in G$, and $a<x[a>x]$ imply $x \in A$. The empty set is denoted by $\varnothing$, and $A \backslash B$ means the set of elements of $A$ not in $B$.

By the order dual $G^{*}$ of $G$ we mean the group $G$ endowed with the dual $\varliminf^{*}$ of $\leqq(a \leqq * b \Leftrightarrow b \leqq a)$. $G$ and $G^{*}$ have the same sets of left [right] conservers and inverters.

Theorem 1. Let $G$ be a po-group of the second kind. Let $H[I]$ be the set of conservers [inverters] of $G$. Then $H$ is a subgroup of $G$ of index 2, and $I$ is its other coset. $H$ is a po-group of the first kind, and $H_{+}$is normal in $G$. $H$ and $I$ are convex subsets of $G$, and, by passing to the order dual of $G$ if necessary, we can assume that $H$ is an upper class and $I$ a lower class in $G$. In particular, $H_{+}=G_{+}, I_{+}=\varnothing$, and $I_{-}$is a lower class in $G$. The set $I_{-}$has the following properties:

(N1) $I_{-}$is normal in $G$.

(N2) $I_{-}^{-1}=I_{-}$.

(N3) $I_{-}$contains $H_{+} I_{-}, I_{-} H_{+}, H_{-} I_{-}$, and $I_{-} H_{-}$.

The order relation $\leqq$ can be described in terms of $H_{+}$and $I_{-}$as follows:

(O1) If $x \in H, y \in H$, then $x \leqq y \Leftrightarrow x^{-1} y$ (or $\left.y x^{-1}\right) \in H_{+}$.

(O2) If $x \in I, y \in I$, then $x \leqq y \Leftrightarrow x y^{-1}$ (or $\left.y^{-1} x\right) \in H_{+}$.

(O3) If $x \in I, y \in H$, then $x \leqq y \Leftrightarrow x y^{-1}$ (or $y^{-1} x$ or $x^{-1} y$ or $\left.y x^{-1}\right) \in I_{-}$. 
(O4) If $x \in H, y \in I$, then $x \leqq y$ never holds.

If $H$ is directed, then $I_{-}$must be I or $\varnothing$. If $I_{-}=I$, then every element of $I$ is less than every element of $H$. If $I_{-}=\varnothing$, then no element of $I$ is comparable with any element of $H$.

Conversely, let $G$ be a group containing a subgroup $H$ of index 2, and let $I=G \backslash H$. Assume that $H$ is a po-group of the first kind, and that its positive cone $H_{+}$is normal in $G$. Let $I_{-}$be a subset of $I$ having properties (N1-3). Define $\leqq$ in $G$ by (O1-4). This agrees with the given partial order in $H$ by (O1), and $G$ becomes thereby a po-group of the second kind such that $H[I]$ is the set of conservers [inverters] of $G$.

Remarks. (1) If (N1) and (N2) hold, and if $I_{-}$contains any one of the four product sets in (N3), then it contains the other three.

(2) Regarding the parenthetical assertions in (O3), we note that if $I_{-}$is any subset of $I$ satisfying (N1) and (N2), and if any one of the four products $x y^{-1}, y^{-1} x, x^{-1} y, y x^{-1}$ belong to $I_{-}$, then so do the remaining three. A similar remark applies to $(\mathrm{O} 1)$ and $(\mathrm{O} 2)$, since $H_{+}$is normal in $G$.

PROof. Evidently the product of two conservers or of two inverters is a conserver, while that of a conserver and an inverter is an inverter. Since the identity element $e$ of $G$ is a conserver, the inverse of a conserver [inverter] must be of the same type. From these remarks it is clear that $H$ is a subgroup of $G$ of index 2 , that $I=G \backslash H$, and that $H$ is a po-group of the first kind.

If $p \in H_{+}$and $u \in I$, then from $e \leqq p$ we have $u \geqq p u$ and $e=u^{-1} u \leqq u^{-1} p u$. Thus $u^{-1} H_{+} u \subseteq H_{+}$. Since $H_{+}$is normal in $H$, this shows that it is normal in $G$.

To show that $H$ is convex in $G$, it clearly suffices to show that $e<u<h(h \in H, u \in I)$ is impossible. Multiplying $e<u<h$ on the left by the inverter $u$, and on the right by the conserver $h$, we obtain $u>u^{2}>u h$ and $h<u h<h^{2}$. But this yields $u>u h>h$, contrary to $u<h$.

To show that $I$ is convex in $G$, suppose that $u>h>u^{\prime}(h \in H$; $\left.u, u^{\prime} \in I\right)$. Then $e<h u^{-1}<u^{\prime} u^{-1}$. Since $u^{\prime} u^{-1} \in H$ and $h u^{-1} \in I$, this contradicts the convexity of $H$.

From $G=H \cup I$ it follows that $H$ must be either an upper class or a lower class in $G$. By passing to the order dual of $G$, if necessary, we can assume that $H$ is an upper class. Then $I$ is a lower class. Since $e \in H$, we have $G_{+} \subseteq H$, and hence $H_{+}=G_{+}$and $I_{+}=\varnothing$. $I_{-}$is clearly a lower class in $I$, hence also in $G$.

If $u \in I_{-}$and $v \in I$, then from $u<e$ we have $u v>v$ and $v^{-1} u v<v^{-1} v=e$, hence $v^{-1} u v \in I_{-}$. Similarly, $h^{-1} u h \in I_{-}$for every $h$ in $H$, which proves (N1). To show (N2), we note that $u<e(u \in I)$ implies $e=u u^{-1}>e u^{-1}=u^{-1}$, hence $u^{-1} \in I_{-}$. By Remark (1), to estab- 
lish (N3) we need only show that $H_{+} I_{-} \subseteq I_{-}$. From $h>e, u<e(h \in H$, $u \in I)$, we have $h u<e u=u<e$, so that $h u \in I_{-}$.

(O1) is a standard fact about po-groups, and (O4) is just the assertion that $H$ is an upper class in $G$. To show (O2), we note that $x \leqq y$ is equivalent to $x y^{-1} \geqq e$, since $y \in I$. To show (O3), we observe that $x \leqq y$ is now equivalent to $x y^{-1} \leqq y y^{-1}=e$, since $y \in H$.

If $H$ is directed, then $H=H_{+} H_{-}$, and (N3) implies that $H I_{-} \subseteq I_{-}$. If $I_{-} \neq \varnothing$, let $u \in I_{-}$. Then $I=H u \subseteq I_{-}$, whence $I_{-}=I$.

Turning to the converse, let $H$ be a subgroup of $G$ of index 2, and let $H$ be a po-group of the first kind such that $H_{+}$is normal in $G$. Let $I=G \backslash H$. Let $I_{-}$be a subset of $I$ having properties (N1-3), and define $\leqq$ in $G$ by $(\mathrm{O} 1-4)$. (O1) asserts that the restriction of $\leqq$ to $H$ shall coincide with the given partial ordering of $H$.

That the relation $\leqq$ is reflexive and antisymmetric is clear. To prove that it is transitive, let $x \leqq y$ and $y \leqq z(x, y, z \in G)$. (O4) implies that if $x \in H$ then $y \in H$, and if $y \in H$ then $z \in H$. Since we do not need to consider the case $x, y, z \in H$, we are left with three cases.

Case $x \in I, y \in H, z \in H$. By (O3) and (O1) we have $x^{-1} y \in I_{-}$and $y^{-1} z \in H_{+}$. By (N3), $x^{-1} z=\left(x^{-1} y\right)\left(y^{-1} z\right) \in I_{-}$, and $x \leqq z$ by (O3).

Case $x \in I, y \in I, z \in H$. By (O2) and (O3) we have $x y^{-1} \in H_{+}$and $y z^{-1} \in I_{-}$. By (N3), $x z^{-1}=\left(x y^{-1}\right)\left(y z^{-1}\right) \in I_{-}$, and $x \leqq z$ by (O3).

Case $x \in I, y \in I, z \in I$. By (O2) we have $x y^{-1} \in H_{+}$and $y z^{-1} \in H_{+}$. Hence $x z^{-1}=\left(x y^{-1}\right)\left(y z^{-1}\right) \in H_{+}$, and $x \leqq z$ by (O2).

Hence $G$ is a po-set under $\leqq$. All that remains is to show that every element of $H[I]$ is a conserver [inverter]. Since every element of $H$ is the product of two elements of $I$, it suffices to show that every element of $I$ is an inverter.

Let $u \in I$, and let $x \leqq y$. The case $x \in H, y \in I$ is excluded by (O4), and we consider the remaining three.

Case $x \in H, y \in H$. By (O1), $x^{-1} y$ and $y x^{-1} \in H_{+}$. Hence $(u x)^{-1}(u y)$ and $(y u)(x u)^{-1} \in H_{+}$, and we infer from (O2) that $u y \leqq u x$ and $y u \leqq x u$.

Case $x \in I, y \in I$. By $(\mathrm{O} 2), x y^{-1}$ and $y^{-1} x \in H_{+}$. Hence $(x u)(y u)^{-1}$ and $(u y)^{-1}(u x) \in H_{+}$, and we infer from (O1) that $y u \leqq x u$ and $u y \leqq u x$.

Case $x \in I, y \in H$. By (O3), $x^{-1} y$ and $y x^{-1} \in I_{-}$. Hence $(u x)^{-1}(u y)$ and $(y u)(x u)^{-1} \in I_{-}$, and we infer from (O3) that $u y \leqq u x$ and $y u \leqq x u$.

This concludes the proof of the theorem.

Let us consider all possible ways of extending a given po-group $H$ of the first kind to a po-group $G$ of the second kind, such that $H$ is the set of conservers of $G$. In the first place, $G$ must be an extension of $H$ by the cyclic group $C_{2}$ of order 2; the Schreier theory tells us how to find all such. Call $G$ "suitable" if $H_{+}$is normal in $G$; there is at 
least one suitable $G$, namely the direct product $H \times C_{2}$. Any suitable $G$ can be partially ordered in the desired fashion by choosing $I_{-}$so as to satisfy (N1-3). This can always be done by choosing $I$ or $\varnothing$ for $I_{-}$, and these are the only possibilities if $H$ is directed. If $G$ itself is to be directed, only $I_{-}=I$ is possible, and then every element of $H$ exceeds every element of $I$. In this case we note that $G$ will be latticeordered or totally ordered if and only if the same holds for $H$.

If $H$ is trivially ordered, then $I_{-} \neq \varnothing$, since $G$ cannot be trivially ordered. As a simple example with $I_{-} \neq I$, let $G$ be the infinite cyclic group generated by $a$, let $H$ be the subgroup generated by $a^{2}$, and let $I_{-}=\left\{a, a^{-1}\right\}$. The resulting partial order on $G$ has a saw-tooth nature:

$$
\ldots>a^{-3}<a^{-2}>a^{-1}<e>a<a^{2}>a^{3}<\cdots
$$

3. Partially ordered groups of the third kind. We define the following four subsets of a po-group $G$ of the third kind. Let $C_{2}=\{0,1\}$ be the additive group of integers $\bmod 2$, so that $1+1=0$. For $i$ and $j$ in $C_{2}$ let $G_{i j}$ be the set of all elements $a$ of $G$ such that $a$ is a left conserver if $i=0$, a left inverter if $i=1$, a right conserver if $j=0$, and a right inverter if $j=1$.

From the way left and right conservers and inverters multiply,

$$
G_{i j} G_{k l}=G_{i+k, j+l} \quad\left(i, j, k, l \in C_{2}\right) .
$$

By definition of po-group of the third kind,

$$
G=G_{00} \cup G_{01} \cup G_{10} \cup G_{11}, \quad G_{01} \cup G_{10} \neq \varnothing .
$$

If $G_{01}$ and $G_{10}$ are $\neq \varnothing$, then $G_{11} \neq \varnothing$.

THEOREM 2. Let $G$ be a po-group of the third kind, with $G_{i j}$ as defined above. Then $G_{00}$ is a normal subgroup of $G$, and is a po-group of the first kind. If $G_{11}=\varnothing$, then $G / G_{00} \cong C_{2}$. If $G_{11} \neq \varnothing$, then $G / G_{00} \cong C_{2} \times C_{2}$, and $G_{00} \cup G_{11}$ is a normal po-subgroup of $G$ of the second kind. The positive cone $P$ of $G_{00}$ satisfies the following conditions:

$\left(\mathrm{N}^{\prime} 1\right)$ if $a \in G_{00} \cup G_{11}$, then $a P a^{-1} \subseteq P$;

$\left(\mathrm{N}^{\prime} 2\right)$ if $a \in G_{01} \cup G_{10}$, then $a P a^{-1} \subseteq P^{-1}$.

No two elements belonging to different $G_{i j}$ are comparable. Within each $G_{i j}$, the order relation is given in terms of $P$ as follows:

$\left(\mathrm{O}^{\prime} 1\right)$ if $x, y \in G_{00}$ or $x, y \in G_{01}$, then $x \leqq y \Leftrightarrow x^{-1} y \in P$;

$\left(\mathrm{O}^{\prime} 2\right)$ if $x, y \in G_{10}$ or $x, y \in G_{11}$, then $x \leqq y \Leftrightarrow x^{-1} y \in P^{-1}$.

Conversely, let $G$ be a group containing a normal subgroup $H_{00}$ such that $G / H_{00} \cong C_{2}$ or $C_{2} \times C_{2}$. In the latter event, let $H_{i j}$ be the coset of $H_{00}$ in $G$ mapped into the element $(i, j)$ of $C_{2} \times C_{2}$. Assume that $H_{00}$ is a 
po-group of the first kind such that its positive part $P$ satisfies $\left(N^{\prime} 1-2\right)$, with $G_{i j}$ replaced by $H_{i j}$. Define a relation $\leqq$ on $G$ by $\left(\mathrm{O}^{\prime} 1-2\right)$, similarly modified, with $\leqq$ never holding between elements of distinct $H_{i j}$. Then $G$ becomes a po-group of the third kind, with $G_{i j}=H_{i j}$. The same holds in the event $G / H_{00} \cong C_{2}$ if we let $H_{11}=\varnothing$, and either $H_{01}=\varnothing$ or $H_{10}=\varnothing$.

Proof. The first three sentences are obvious. $\left(N^{\prime} 1\right)$ then follows from Theorem 1. To show ( $\left.\mathrm{N}^{\prime} 2\right)$, let $p \in P$ and let $a \in G_{01}$. From $e<p$ we have $a<a p$, since $a$ is a left conserver, and hence $e>a p a^{-1}$, since $a$ is a right inverter. Thus $a p a^{-1} \in P^{-1}$. The proof for $a$ in $G_{10}$ is similar.

We note that the identity element $e$ of $G$ cannot be comparable with any element of $G_{01}$ or $G_{10}$. For if $e<a\left(a \in G_{01}\right)$, then $a<a^{2}$ since $a$ is a left conserver, and $a>a^{2}$ since $a$ is a right inverter. The argument is similar if $e>a$, or if $a \in G_{10}$. Moreover, $e$ cannot be comparable with an element $a$ of $G_{11}$. For suppose $a<e$. Let $b \in G_{01}$. Then $b a<b$ and $b a b^{-1}>e$, since $b^{-1} \in G_{01}$. But $b a b^{-1} \in G_{01} G_{11} G_{01}=G_{11}$, and $a<e<b a b^{-1}$ would violate the convexity of $I=G_{11}$ in the po-group $G_{00} \cup G_{11}$ of the second kind (Theorem 1). The argument is similar if $e<a$.

Now let $a$ and $b$ be any two elements of $G$ such that $a<b$. Then $a a^{-1}<b a^{-1}$ or $a a^{-1}>b a^{-1}$, depending on whether $a^{-1}$ is a right conserver or a right inverter. In either case, $b a^{-1}$ is comparable with $e$, and so belongs to $G_{00}$. Hence $a$ and $b$ belong to the same coset $G_{i j}$.

To show $\left(\mathrm{O}^{\prime} 1\right), x \leqq y \Leftrightarrow e \leqq x^{-1} y \Leftrightarrow x^{-1} y \in P$, since $x$ is a left conserver. As for $\left(\mathrm{O}^{\prime} 2\right), x$ is a left inverter, and so $x \leqq y \Leftrightarrow e \geqq x^{-1} y \Leftrightarrow x^{-1} y \in P^{-1}$.

Proceeding to the converse, let us introduce the notation $P_{0}=P$, $P_{1}=P^{-1}$, where $0,1 \in C_{2}$. Then, for any $k$ in $C_{2}, P_{k}^{-1}=P_{k+1}$. The modified rules $\left(\mathrm{N}^{\prime} 1-2\right)$ and $\left(\mathrm{O}^{\prime} 1-2\right)$ can then be condensed into single formulae:

$\left(\mathrm{N}^{\prime}\right)$ if $a \in H_{i j}$, then $a P a^{-1} \subseteq P_{i+j}$;

$\left(\mathrm{O}^{\prime}\right)$ if $x, y \in H_{k l}$, then $x \leqq y \Leftrightarrow x^{-1} y \in P_{k}$.

It is evident that $\leqq$ defined by $\left(\mathrm{O}^{\prime}\right)$ is reflexive and symmetric. If $x \leqq y$ and $y \leqq z$, then $x, y$, and $z$ all belong to the same $H_{k l}$, and $x^{-1} z=\left(x^{-1} y\right)\left(y^{-1} z\right) \in P_{k} P_{k} \subseteq P_{k}$, whence $\leqq$ is transitive.

To show that $H_{i j}=G_{i j}$, let $a \in H_{i j}$ and let $x \leqq y$. Then $x$ and $y$ belong to the same $H_{k l}$ and $x^{-1} y \in P_{k}$. From $(a x)^{-1}(a y)=x^{-1} y \in P_{k}$, and $a x$, $a y \in H_{i+k, j+l},\left(\mathrm{O}^{\prime}\right)$ gives $a x \leqq a y$ if $i=0$ and $a y \leqq a x$ if $i=1$. Since this is independent of $k$ and $l$, we conclude that $a$ is a left conserver if $i=0$ and a left inverter if $i=1$.

From $(x a)^{-1}(y a)=a^{-1}\left(x^{-1} y\right) a \in P_{k+(i+j)}=P_{(i+k)+j}$ by $\quad\left(\mathrm{N}^{\prime}\right)$, and $x a, y a \in H_{i+k, j+l}$, we conclude from $\left(\mathrm{O}^{\prime}\right)$ that $x a \leqq y a$ if $j=0$ and $y a \leqq x a$ if $j=1$. Hence $a$ is a right conserver if $j=0$ and a right inverter if $j=1$. 
Let $H$ be a po-group of the first kind. We saw at the conclusion of $\S 2$ that $H$ can be extended in at least one way to a po-group $G$ of the second kind. This is not so if $G$ is to be of the third kind. In fact it is possible if and only if there exists an automorphism of $H$ the square of which is inner, and which maps the positive cone $P$ of $H$ into $P^{-1}$. This is always possible if $H$ is abelian, since $x \rightarrow x^{-1}$ is then an automorphism with these properties. But it is impossible if $H$ is a group every automorphism of which is inner. For example, let $H$ be the group of rational matrices of the form

$$
\left(\begin{array}{ll}
a & b \\
0 & 1
\end{array}\right)
$$

with $a>0$, and define $P(H)$ to be the set of all such matrices with $a \geqq 1$.

\section{REFERENCES}

1. J. A. H. Shepperd, Betweenness groups, J. London Math. Soc. 32 (1957), 277285.

2. A. H. Clifford, Ordered commutative semigroups of the second kind, Proc. Amer. Math. Soc. 9 (1958), 682-687.

3. J. Gilder, Betweenness and order in semigroups, Proc. Cambridge Philos. Soc. 61 (1965), 13-28.

4. K. Keimel, Halbgruppen mit Zwischenbedingung, Staatsexamensarbeit, Tübingen, Germany, 1964.

5. J. F. Andrus and A. T. Butson, Ordered groups, Amer. Math. Monthly 70 (1963), 619-628.

Newcomb College, Tulane University 